\title{
Komplementärmedizinische Beratung in der Onkologie
}

\author{
Matthias Rostock $^{\mathrm{a}, \mathrm{b}} \quad$ Reinhard Saller ${ }^{\mathrm{a}}$ \\ a Institut für Naturheilkunde, Universitätsspital Zürich, Schweiz \\ ${ }^{\mathrm{b}}$ Universitäres Cancer Center Hamburg, Hubertus Wald Tumorzentrum, Universitätsklinikum Hamburg-Eppendorf, Deutschland
}

In den letzten 10-15 Jahren sind im deutschen Sprachraum an verschiedenen Universitätskliniken Beratungsmöglichkeiten für Krebspatienten zu komplementärmedizinischen Therapieoptionen eingerichtet worden. Zum Teil sind entsprechende Experten direkt in den universitären Krebszentren beschäftigt wie z.B. in Jena, Hamburg, Frankfurt/M. oder Zürich, wo das Institut für Naturheilkunde in den Bereich Innere Medizin Onkologie integriert ist. Zum Teil sind konsiliarische Kooperationen etabliert wie in Berlin zwischen dem Cancer Center der Charité und der CHAMP-Ambulanz am Institut für Sozialmedizin, Epidemiologie und Gesundheitsökonomie, in Freiburg i.Br. mit dem Unizentrum Naturheilkunde, in München mit dem Kompetenzzentrum Komplementärmedizin Naturheilkunde (KoKoNat), in Köln mit dem Institut zur wissenschaftlichen Evaluation naturheilkundlicher Verfahren oder in Bern mit der Kollegialen Instanz Komplementärmedizin (KIKOM). In anderen Universitätskliniken sind entsprechende Einrichtungen in anderen Abteilungen etabliert, insbesondere in der Gynäkologie, z.B. in Heidelberg, in Gießen, oder in Wien. In Essen gibt es eine Kooperation zwischen dem Brustzentrum und der Klinik für Integrative Medizin und Naturheilkunde. An der Berliner Charité und am Haunerschen Kinderspital in München befinden sich entsprechende Einrichtungen in der Pädiatrie bzw. der pädiatrischen Onkologie, an der Medizinischen Hochschule Hannover in der Strahlentherapie.

An das therapeutische Handeln und die therapeutische Beratung innerhalb universitärer Einrichtungen wird der Anspruch einer wissenschaftlichen Nachprüfbarkeit gestellt - für viele heißt dies, dass nur das, was gemäß der sogenannten, allerdings oft missverstandenen und verkürzt wahrgenommenen Evidence-Based Medicine in seiner Wirksamkeit belegt ist, auch zur Anwendung kommen sollte. Erfreulicherweise hat das Forschungsvolumen in der komplementärmedizinischen Onkologie in den letzten Jahren deutlich zugenommen, vor allem aufgrund einer endlich zunehmenden Verfügbarkeit der Forschungsmittel. Zu einigen Therapieverfahren finden sich in der hier vorliegenden
Ausgabe von Forschende Komplementärmedizin Übersichtsarbeiten, die den jeweiligen aktuellen Forschungsstand zusammenfassen. So ist es heute zuverlässiger als noch vor wenigen Jahren möglich, eine Reihe komplementärmedizinischer Patientenfragen entsprechend dem Forschungsstand aufzugreifen.

Nichtsdestotrotz ist die Zahl der wissenschaftlich überprüften komplementären Therapien in der Onkologie nach wie vor begrenzt. Man steht immer wieder vor der Frage, wie ein sinnvoller Umgang mit möglicherweise hilfreichen, aber wissenschaftlich nicht genügend evaluierten naturheilkundlichen bzw. komplementärmedizinischen Therapieansätzen aussehen könnte. Als anschauliches Beispiel hierfür mag der Beitrag zu antiphlogistisch wirksamen Phytotherapeutika [1] dienen. In einer Reihe nichtuniversitärer Einrichtungen und in vielen Praxen scheint durchaus eine relativ große therapeutische Experimentierfreude $\mathrm{zu}$ bestehen, die mitunter aber auch dazu führen kann, dass den Patienten fragwürdige Therapien zu möglicherweise hohen Kosten angeboten werden. In universitären Beratungsambulanzen herrscht dagegen häufig die Maxime vor, dass von der Anwendung einer Methode oder eines Arzneimittels, für das es in der geplanten Indikation keine randomisierten Studien gibt, in der Regel abgeraten werden sollte.

Ungelöst ist weiterhin die Frage, durch welche begründete Einstellung der beratende Arzt dem einzelnen Patienten mit komplementärmedizinischen Fragen am ehesten gerecht werden kann. Dabei sollte primär das Anliegen des Patienten im Fokus stehen, und nicht allein das Diktat der wissenschaftlichen Überprüfbarkeit. Das Anliegen unterschiedlicher Patienten an ihren komplementärmedizinisch beratenden Arzt kann in vergleichbaren Therapiesituationen sehr unterschiedlich sein. Eine kanadische Arbeitsgruppe um Lynda G. Balneaves und Marja Verhoef [2] arbeitete im Rahmen einer qualitativen Studie mit Brustkrebspatientinnen drei verschiedene Formen der Entscheidungsfindung bezüglich komplementärmedizinischer Therapien im therapeutischen Gesamtkonzept ihrer Tumorerkrankung heraus:

\section{KARGER \\ Fax +497614520714 \\ Information@Karger.de}

www.karger.com (c) 2011 S. Karger GmbH, Freiburg

$1661-4119 / 11 / 0184-0174 \$ 38.00 / 0$

Accessible online at:

www.karger.com/fok
Dr. Matthias Rostock

Institur für Naturheilkunde

Universitätsspital Zürich

Rämistrasse 100, 8091 Zürich, Schweiz

matthias.rostock@usz.ch 
- In der ersten Gruppe («Taking it one step at a time») werden die Patientinnen zusammengefasst, die eine hohe Erwartungshaltung sowohl gegenüber der Komplementärund Alternativmedizin (CAM) als auch gegenüber der konventionellen Therapie haben. Widersprüchliche Informationen, die sie möglicherweise von Seiten des Onkologen und des CAM-Therapeuten bekommen, führen $\mathrm{zu}$ einem erheblichen Stress und zu intensivem Klärungsbedarf in der komplementärmedizinischen Beratung. Aus Sorge vor möglichen Interaktionen neigen diese Patientinnen eher dazu, während der konventionellen onkologischen Behandlung auf pharmakologische komplementärmedizinische Therapien gänzlich zu verzichten, während weniger invasive Anwendungen wie Meditation oder Reiki eher beibehalten werden. Erst nach Abschluss der konventionellen Therapie wenden sich diese Patientinnen dann wieder vermehrt auch medikamentösen komplementärmedizinischen Therapien zu.

- Patientinnen der zweiten Gruppe («Playing it safe») vertrauen in erster Linie auf die konventionelle Therapie und auf Ergebnisse wissenschaftlicher Studien aus anerkannten medizinischen Zeitschriften. Für diese Patientinnen kommt eine komplementäre Therapie nur in Frage, wenn es hierzu Ergebnisse aus kontrollierten klinischen Studien gibt. An darüber hinaus gehenden Informationen besteht im Allgemeinen kein großes Interesse.

- Zu der dritten Gruppe («Bringing it all together») werden gemäß der Untersuchung Patientinnen gezählt, die im Vorfeld ihrer Erkrankung bereits seit langem CAM-Verfahren angewendet hatten. Für diese Patientinnen gehört auch die Komplementärmedizin bei der Behandlung ihrer Krebserkrankung mit großer Selbstverständlichkeit dazu. Sie suchen sich Onkologen, die CAM-Verfahren gegenüber offen sind. Wenn es dennoch zu widersprüchlichen Aussagen von behandelnden Onkologen und Komplementärmedizinern kommt, verfolgen sie in Ruhe ihren eingeschlagenen, oft auf eigener Intuition beruhenden Weg.

Die Ergebnisse dieser Untersuchung bestätigen die Erfahrungen aus der täglichen Praxis, dass sehr viele Patienten mit schablonenhafter Beratung - auch wenn sie auf dem jeweils aktuellsten Stand der relevanten Forschung beruht - in der Regel nicht zufrieden sind und dass die Beratungsarbeit neben onkologischer und komplementärmedizinischer Kompetenz auch ein hohes Maß an Patientenzentriertheit erfordert, um zu ergründen, wo die wesentlichen Bedürfnisse des Patienten liegen und wie und womit diesen am ehesten entsprochen werden kann [3]. Hierfür ist es auch notwendig, mit Offenheit und ohne Vorurteile die Werte und Vorstellungen des Patienten bezüglich seiner Erkrankung und der von ihm mehr oder weniger favorisierten Therapieansätze zu erforschen. Ein Patient, der sich diesbezüglich nicht gesehen und respektiert fühlt - unabhängig davon, ob wir ihn in seiner Sichtweise aus verantwortlich-beratender Position bestätigen können oder nicht -, wird sich schwer tun, einen therapeutischen Rat anzunehmen.

Bereits in seiner ursprünglichen Definition von EvidenceBased-Medicine schloss David Sackett die Patientensicht implizit mit ein: «The integration of the best research evidence with clinical expertise and patient values» [4]. Gerade in der Gruppe der CAM-erfahrenen Patienten treffen wir häufig auf eine sehr skeptische Haltung gegenüber Ärzten, die sich in ihrer Urteilsbildung ausschließlich auf Daten aus wissenschaftlichen Untersuchungen stützen. Stattdessen besteht eine größere Bereitschaft, auch Empirie, Einzelfallberichte, persönliche Erfahrungen anderer Patienten und die eigene Intuition heranzuziehen. In einer qualitativen Untersuchung der Frage, welche Form von Evidenz für Tumorpatienten in ihrer Entscheidungsfindung hinsichtlich komplementärmedizinischer Therapien höherrangig eingeschätzt wird, fanden Verhoef et al. [5] folgerichtig, dass erfahrene CAM-Nutzer eher inneren als äußeren Wissensquellen vertrauten.

Komplementärmedizinische Beratung von tumorkranken Patienten, die ernst genommen wird, ist nicht möglich, ohne dass man sich als Therapeut immer wieder neu einlässt auf das Spannungsfeld zwischen Subjektivität und Objektivität, zwischen nüchternen wissenschaftlichen Daten und motivationsbestimmenden und möglicherweise wirksamkeitsmitbestimmenden Vorstellungswelten, zwischen dem Risiko möglicher Interaktionen mit konventionellen antitumoralen Therapien und der Option auf eine supportive oder auch antitumorale therapeutische Wirksamkeit komplementärmedizinischer Therapieverfahren. Es versteht sich von selbst, dass sich ein komplementärmedizinischer Berater an einer universitären Einrichtung der Wissenschaftlichkeit verpflichtet fühlt. Wenn er dabei in Grenzbereiche bestehenden Wissens vordringt, führt dies nicht zwangsläufig zu therapeutischem Nihilismus, sondern es ist dennoch ein verantwortliches und reflektiertes Handeln möglich, insbesondere wenn Wissen und Nichtwissen sowie Möglichkeiten und Grenzen der Einschätzung bewusst sind und dementsprechend formuliert werden.

\section{Literatur}

1 Saller R, Melzer J, Rostock M: Antiinflammatorisch wirksame Phytotherapeutika und ihr mögliches Potential bei tumorkranken Menschen. Forsch Komplement Med 2011;18:203-212.

2 Balneaves LG, Truant TL, Kelly M, Verhoef MJ, Davison BJ: Bridging the gap: decision-making processes of women with breast cancer using complementary and alternative medicine (CAM). Support Care Cancer 2007;15:973-983.

3 Frenkel M: Clinical consultation, a personal perspective: components of a successful integrative medicine clinical consultation. J Soc Integr Oncol 2008;6: $129-133$.

4 Sackett D, Straus S, Richardson W, Rosenberg W, Haynes RE (eds): EvidenceBased Medicine: How to Practice and Teach EBM. Edinburgh, Churchill Livingstone, 2000 .

5 Verhoef MJ, Mulkins A, Carlson LE, Hilsden RJ, Kania A: Assessing the role of evidence in patients' evaluation of complementary therapies: a quality study. Integr Cancer Ther 2007;6:345-353. 